Departamento de Anatomia Descritiva dos Animais Domésticos Diretor: Prof. M. Barros Frhart

DOIS CASOS DE A. SUBCLAVIA DEXTRA COMO ÚLTIMA COLATERAL DO ARCUS AORTICUS NO CÃO $\left(^{*}\right)$

\author{
(TWO CASES OF A. SUBCLAVIA DEXTRA AS THE LAST BRANCH
} OF THE ARCUS AORTICUS IN THE DOG)

Orlando M. Paiva

1 estamph (2 figuras)

Fez o acaso com que em estudando a disposição dos ramos conspícuos da crossa aórtica no cão, tendo por objetivo a verificação da freqüência do Truncus bicaroticus, se nos deparasse o ensejo de registrar, em dois dos exemplares, a mesma e rara anomalia de origem e de trajeto da $A$. subclavia dextra. Por se tratar de observação deveras pouco comum, no âmbito da literatura anátomo-comparativa, resolveu-se dá-la à publicação antes mesmo que as anotações sôbre a ocorrência do tronco bicarótico, ainda em elaboração, viessem a lume.

Nos carnivoros domésticos - recordemos sucintamente - dois vasos emergem da crossa da aorta em condições normais: $A$. brachiocephalica e $A$. subclavia sinistra; a $A$. brachiocephalica, por sua vez, emite uma após outra as Aa. carotides communes sinistra e dextra, continuando-se como A. subclavia dextra. Contrastando todavia, manifestamente, com a disposição habitual, os exemplares em aprêço exibiam a A. subclavia dextra nascendo justaposta e crânio-distalmente à $A$. subclavia sinistra, tendo além do mais, ao cruzar a linha mediana para atingir o membro torácico correspondente, percurso supra-esofágico. Em conseqüência, o primeiro ramo calibroso a ser fornecido pela crossa da aorta era representado pelo tronco das $A a$. carotides communes sinistra e dextra, seguindo-se, em ordem, a curta distância e distalmente, as origens das Aa. subclaviae sinistra e dextra.

Assim, à particular disposição da $A$. subclavia dextra se acrescia, nos dois espécimes, outra igualmente catalogável como desusada, embora de frequência por suposto mais elevada, ou seja, a ocorrência do Truncus bicaroticus. E, finalmente, ainda um incidente importante, tal como o anterior acarretado pela procedência e decurso inusitados da $A$. subclavia dextra, podia ser verificado em ambos os casos, por isso que, o $N$. laryngeus caudalis, ramo fornecido pelo $N$. vagus em seu trajeto torácico, ao invés de se apresentar com o percurso que

22-28/1/19) Apresentudo ao IV congresso Brasileiro de Medicina Veterinaria, Rio de Janeiro, 
conhecemos e lhe vale também o nome de $N$. recurrens, originava-se da porção cervical clo N. vagus tendo, em consęüência, seu trajeto recorrencial em direção à laringe, sobremaneira reduzido.

Nesta nota apresentamos, pois, minuciosamente descritas, as anomalias de origem e de trajeto da $A$. subclavia dextra e do $N$. laryngeus caudalis, e analisamos as condiçōes embriolúgicas que encaminharam o desenvolvimento de ambas. A presença do tronco bicarótico, como acontecimento diretamente subordinado à origem invulgar da A. subclavia dextra, perde muito do relêvo anatômico de que se reveste em outras circunstâncias e, por tal motivo, não merecerá comentários especiais.

Muito freqüentes, as variações e anomalias das colaterais da crossa aórtica explicam-se pela eventual intercorrência de modificaçōes, determinadas por múltiplos fatores, na série de fenômenos inerentes ao evolver do sistema arterial à altura dos arcos aórticos. Os decorrentes defeitos, quando compatíveis com a vida, revelam-se casualmente e ora se traduzem quer pelo aumento, quer pela diminuição do número dos ramos da crossa da aorta, ora se espelham em irregularidades respeitantes à origem de tais ramos, ou ainda refletem-se em alterações no trajeto dos vasos com quebra das usuais relações anatômicas.

De fato, na literatura anatômica humana contam-se às centenas as notificações de transposição de origem da $A$. subclavia dextra, isto é, de seu nascimento como último ramo do Areus norticus, associada a decurso retro-esofágico do vaso; concomitantemente, descrevemse a origem das Aa. carotides communes por Truncus bicaroticus, em alguns casos e, em quase todos, a não recorrência do $N$. lruryngeus caudalis homolateral. No entanto, e por pouco plausível que pareça, só excepcionalmente se encontram registrados casos, da mesma ou de semelhante natureza, em animais domésticos. Além do mais, os poucos casos de variações dos ramos do arco aúrtico dados à publicação, " foram, quase totalmente, mais pelo interêsse clínico envolvido, quando não resultaram de encontro acidental em exames post-mortem, constituindo, portanto, achados de consultório, de salas de necrópsia e só fortuitamente de mesas anatômicas; essa deve ser, com toda a probabilidade, a razão pela qual a maioria dos $\mathrm{AA}$. emprestou secundária importância ao aspecto morfológico das peças.

A bibliografia anatômica veterinária compulsada ofereceu cêrca de uma dezena de trabalhos dispersos, versando sôbre anomalias do arco aórtico e de seus ramos mais calibrosos no cão doméstico; como veremos, as observações coligidas dizem respeito, em sua maioria, à persistência da aorta primitiva direita ao invés da correspondente es- 
O. M. Paiva - A. subclávia direita como última colateral da aorta no cão 205

querda, citando um dos AA., dois casos de decurso supra-esofágico da A. subclavia sinistra; em tồlas as circunstâncias, todavia, o interêsse precipuo das publicaçōes vinculava-se à obstrução esofágica motivada pelas referidas anomalias.

As únicass observações em mamíferos, relativas à origem inusitada da $A$. sublavia dextra como última colateral aúrtica, são, ao que se sabe, afora as de MECKEL (1810) no ouriço cacheiro (Evinaceus) e de SMITII (1891) no coelho, mencionadas na monografia de HolzapFel (1899), a de EALES (1930) também no coelho. SMITH, aliás, assinalou seu caso em um único exemplar, dentre 700 examinados, como cital CAIRNEY (1925).

Passando a resumir os relatos apresentados pela literatura, desejamos consignar a convicção de que se deve atribuir à falta de pesquisas sistemáticas, por conseguinte, à escassez de contribuições sôbre o comportamento e variedades dos ramos aórticos, em anatomia comparativa, o desconhecimento pouco menos que absoluto de imporlantes aspectos da morfogenia vascular. Com efeito, apesar de ser esta, provàvelmente, a primeira nota a descrever a $A$. subclavia dextra como último ramo da crossa da aorta no cão, tendo aquêle vaso, para ganhar o membro torácico, decurso entre a coluna vertebral, dorsalmente, e o esôfago e traquéia, ventralmente, apenas o fato de ignorarmos a existência de quaisquer apontamentos a propósito, na espécie em estudo, não scria suficiente para autorizar concluíssemos a rigor e com propriedade tratar-se de raríssima anomalia. Entretanto, se preferimos abandonar a aconselhável e cômoda atitude de rescrva quanto ao grau de incidência, deixando a outrem o pronunciamento a respeito da freqüência, à base de mais farta documentação, devêmo-lo em grande parte à informação gentilmente prestada pelo Prof. MaLColm F. Miller, do Departamento de Anatomia do New York State Veterinary College at Cornell University.

Cientes, circunstancialmente, de suas pesquisas respeitantes à revisão do sistema arterial do cão, endereçamos-lhe notícia das nossas observações indagando seu parecer. A resposta fez-se pronta e ao transcrever parte de seu teor, protestamos sincera gratidão ao Prof. Miller, pela inestimável importância do relato com que nos obsequiou:

I was very interested to hear of your findings in the dog. In the sum 500 dissections which I have either made or observed, I have yet to find a case of the right subclavian artery emerging as the last branch of the aortic arch. I think this must be exceedingly rare.

De nossa parte, se nos é impossível calcular com precisão o número de dissecções realizadas ou examinadas ao curso de exercícios práticos, no decorrer dos últimos anos - e só no biênio 1946-1947 reu- 
nimos mais de uma centena de observações, com o propósito de conhecer a frequiência do tronco bicarótico - podemos, pelo menos, afiançar jamais ter deparado, anteriormente, com as anomalias a estudar, nos exemplares destinados ao, preparo de artérias; adiantaremos mesmo, nunca ter ocorrido a qualquer dos colaboradores do Departamento de Anatomia Descritiva, nestes seus quase três lustros de existência, a oportunidade de registrar caso de A. subclávia direita como último ramo da crossa aórtica no cão.

Gortun (in Brandt -- 1925), após necrópsia praticada em Spaniel de 7 meses destinado ao sacrifício, dada a impossibilidade de alimentar-se, descreve dilatação do esôfago na parte cranial do torax, ocasionada pela situação anormal da aorta à direita do esôfago e da traquucia. Esstas duas formaçōes acham-se, em consuqüência, entre a Aorta e A. pulmonalis, ventralmente ao Ligamentum arteriosum, tendo o último comprimido o esôfago e provocado a estenose com obstáculo à passagem de alimento.

JEX-Br,AKE (1926) aponta caso de obstrução do esôfago produzida pela permanência do Ductus arteriosus, em Lurcher (cruzamento Collie $\mathrm{x}$ Greyhound) condenado ao sacrifício. Diagnóstico clínico: estenose esofágica, eventualmente, espasmo da cárdia. O essencial do exame necroscópico expressa-o o A. nos seguintes têrmos: "The arch of the aorta has persisted on the left side - as in birds - instead of on the right, the branches of the arch coming off in the following order: Right carotic. left carotic, right subclavian, left subclavian. The duct. arteriosus passes across the left side of the oesophagus from pulmonary artery to the aorta, at the origin of the left subclavian, forming a cord $20 \mathrm{~mm}$ long and $5 \mathrm{~mm}$ in thickness." Neste ponto o esôfago é reduzido a cordão de $10 \mathrm{~mm}$ de espessura com lume de $3.5 \mathrm{~mm}$ de diâmetro. Acima da constriçăo mostrava-se n esôfago fortpmente dilatado. Na discussũo realç̧ o $\mathrm{A}$. que o arco aórtico e o Ductus arteriosis, nestes casos, formam anel an redor da traquéia e esôfago, enquanto, normalmente êles se situam do mesmo lado da traquéia e do esôfago. Provàvelmente, trata-se de anomalia semelhante à apontada por GorToN.

Milks (1929) encontra e relata caso de divertículo do esofago. cm cão bastardo, devido à constrição daquêle órgão entre o louctus artẹiosus, dorsalmente, e a A. pulmonar e aorta.

EALES (1930) refere caso de emergência da A. subclávia dircita, como último ramo da crossa aórtica, no coclho. A aorta, como ilustra a $\Lambda$., dá o par das Aa. carótidas comuns, inflete-se para a esquerda dando então saida à A. subclávia esquerda. Distalmente a esta, mas do contórno opôsto, tem origem a A. subclávia direita que, em caminho para o membro direito, se insinua dorsalmente à traquéia e esôfago. $O \mathrm{~N}$. vago e seu ramo (posterior) recorrente laríngeo têm relaçōes normais do lado esquerdo. No lado direito, todavia, o N. recorrente laríngeo se inicia cranialmente à bifurcação das carótidas e não gira ao redor da A. subclávia direita, como faria normalmente, porém, passa adiante c paralelamente à traquéia em direçāo à laringe. Ramo cardíaco é fornecido ao nível da curvatura. 
O. M. Paiva - A. subclávia direita como última colateral da aorta no cão 207

A condição descrita, segundo a A., deve ter tido origem cêrca do $12 .^{\circ}$ dia de desenvolvimento embrionário, conseguintemente a distúrbios dos arcos arteriais. Parece ter havido inibição do crescimento da metade direila do 4. arco aórtico anteriormente ao desenvolvimento do brôto do membro torácico. A A. desconhece a razâo du inibição. Quando, ulteriormente, o membro se formou, um ramo do arco aórtico, distalmente à A. subclávia esquerda e do lado contráyis da aorta, surgiu para supri-lo. O deslocamento caudal do roraçĩo e a alongamento do pescoço, durante o desenvolvimentu, conclue a A., produziram o alongamento do $\mathrm{N}$. laringeo recorrente esquerdo, pois êste passá conlre os arcos aórtico c pulmonar, distalmente ao Dnetres usteriosus. A dileita o $\mathrm{N}$. homónimo formou-se, provàvelmente, antes do movimento caudal do coração e do desenvolvimento da $A$. subclívia anormal. Destarte - N. recorrente é livre e é inusitadamente fornecido alto na região cervical uma anomalia acarretou a outra.

Yananoto e Enoto (in BRANDT - 1935) registram casos de estenuse esofágica em cāo Pastor jovem, morto cêrea dos 4 meses de idade. A compressāo do esôfago e da traquéia era produzida pelo anvolvimento de ambos entre A. pulmonalis, Duetus Botalli e Aorta.

Miloks e Williams (1935) apresentam quatro casos de divertículo esofágico determinado pela constrị̧āo do esôfago, entre Ductus arteriosus. dorsalmente, A. pulmonar e aorta, em 3 Boston-terriers e 1 mestiço. Acrescentando a éstes o caso descrito por um dos A. (MuKs), baseados portanto cm cinco observaçóes, os AA. admitem que sérias aberraçōes no desenvolvimento da anrta no cão, não devem ser extremamente raras, podendo atrihuir-se ì falta de atenção, a virtual ausência de dados sôbre sua ocorrência. Demonstrando, cquando menos, desconhecer os trabalhos de Gorton e JExBLAKE, afirmaßn nāo terem encontrado na literatura anatômica veterinária, no câo ou em outro animal doméstico, qualquer referência a êste defeito embriológico, cuja interpretação reșide na persistêneia da aorta primitiva direita ao invés da esquerda, para constituir a aorta permanente.

Nos casos teratológicos o ducto arterioso origina-se embriològricamente no lado esquerdo. enquanto, a aorta primitiva persiste do lado direito. Conseguintemente o ducto arterioso deve passar sôbre o esôfaggo e a traquéia, afin de atingir a aorta situada anormalmente.

Os AA. discorrem ainda sôbre outro atraente aspecto do problema. ou seja, o da hereditaricdade dos defeitos embriológicos; três dos casos anotados provinham do mesmo "kennel" e dois dêles correspondiam a animais filhos do mesmo macho.

TORÖK (in BRANinT 1938) e Höcsy (1939), éste em dois excmplares, comunicam ohservaçōes de estenose do esôfago, em cão, motivadas pela constriçăo do esôfago torácico e traquéia, entre aorta, A. pulmonar e ligamento arterioso.

Durante a vida fetal, esclarece Möcsy, e imediatamente apús o nascimento, enquanto o ducto arterioso é ainda funcional, haveria espaço apreciável entre as formaçoes arteriais: mas, assim que o ligamento se formou - começou a contrair-se, aumentou a pressão sôbre o esófago e os sintomas de disfagia apareceram. Sintomas de dispnéia não foram notados devido à naturezal mais resistente das paredes da traqucija. 
BrañT (1940) assinala dois casos de arco aórtico direito no cão. L'm ambas as peças a aorta se inicia normalmente do coração, mas dirige-se a seguir para a direita do esôfago c da traquéia, acima do brônquio direito, orientando-se em direção à coluna vertebral e, por fim, caudalmente, algo à direita do plano mediano. Aproximadamente na metado da crossa aórtica origina-se a $A$. brachiocephalica que decorre cranialmente, à direita do esôfago e da traquéia. Na passagem entre crossa aúrtica e aorta descendente, emerge a $A$. subclavia sinistra. Esta artéria corre entĩo em linha reta para a esqucrda e para cima, contudo, cruza dorsalmente o esôfago.

A disposição foi verificada numa fêmea da raça p'astor, de (' semanas e num Bull-dog de 2 anos e meio; o primeiro dos animais apresentava sintomas de constrição do esôfago, enquanto o segundo não os mostrava; a alteração foi descoberta, acidentalmente, durante a rotina de necrópsias.

o A. considera as anomalias mencionadas muito raras, nảo havendo casos tais descritos na literatura técnica publicada; oferece, baseado em JoRES (1924) e G.IRIANI, esclarccimentos a propósito da maneira como se astabelecem as anomalias ao curso do desenvolvimento embrionário.

Com a persistência da $4 . a$ artéria branquial e do tracto de união lateral entre esta e a $6{ }^{\mathrm{a}}$ artéria branquial, em continuação com a raiz aórtica direita, e, com a obliteração das correspondentes formaçōes à esquerda, chega-se uo arco aórtico situado à direita, transcreve Brandr. Permanecendo aberta a raiz ascendente da aorta e a $4 .{ }^{\mathrm{a}}$ e $60^{\mathrm{a}}$ Aa. branqujais esquerdas, então a $A$. subclavia sinistra originar-se-á do Ductus arteriosus (Jones). Suceda o mesmo sómente à raiz aórtica esquerda e a $A$. subclavia sinistra passará a partir da extremidade do Arcus ou início da Aorta descendens. Involuindo, totalmente, a raiz aórtica esquerda ascendente e descendente e mais o $40^{\circ}$ e $6 .^{\circ}$ arcos aórticos esquerdos, ter-se-á a $A$. subclavia sinistra com origem por complemento anormal.

Em preparado remetido ao A., constando de esôfago e estômago, encontrou BRañt a parte anterior do esôfago muito dilatada; caudalmente, a dilatação era fortemente demarcada por estrangulamento, cujo diâmetro media $9 \mathrm{~mm}$; além constrição, o esôfago apresentava largura normal. Como a necrónsia não foi realizada pelo A., é-lhe impossível ajuizar da situaçāo do arco aórtico. Tratando-se, todavia, de animal da mesma raça e idade indicados no caso 1 , situando-se, além disso, a esterose no mesmo ponto, admite a possibilidade de que o animal tenha sido portador de arco aórtico direito.

Davies e OtTAWAy (1934) estudam em colaloração, peculiar caso de obstruçāo do esôfago no cão, em virtude de anomalia congênita do arco aórtico. O animal, Greyhound de 3 meses, encontrava dificuldade em deglutir alimento. A necrópsia demonstrou que, embora não alterando, aparentemente, a distribuiçāo do sangue através do corpo, o desenvolvimento anormal do arco aórtico prejudicou, sèriamente, o esôfago e traquéia. Fstas duas formaçoes, an invés de percorrer'm o mediastino anterior, dorsalmente ao tronco braquiocefálico e à buse do coração, ao lado direito da aorta, como o fazem normalmente, passavam abaixo do troneo brequiocefúlico anormal bicarotico e A. axilar diceita - sôbre a base do coração e A. pulmonar, à esquerda da aorta, para então caminharem entre a dorta e ligancentum urteriosum. Ambas eran, por isso, cncarceradas em cilculo arterial forma. do pelo ligamento arterioso. A pulmonar e aorta. 
0. M. Paiva - A. subclávia direita como última colatera! da sorta no cão 209

Não havia cvidência de constriçāo da traquéia; notava-se, contudo, marcado cstrcitamento do esôfago, neste ponto. Caudalmente à constrição o lume cra normal, porém, cranialmente a cla, na cavidade mediastinal anterior, portanto, existia desenvolvido divertículo onde se acumulava alimento, para, gradualmente, ganhar o estômago, agindo o divertículo como reservatório semelhante ao Ingluvics de aves.

Na discussão anatômica do caso, comparam os AA. as condições descritas com a disposição normal. No espécime portador da obstrução, a primeira artéria a originar-se da uorta, a $2.5 \mathrm{~mm}$ do coração, é o curto tronco hicarótico; ête divide-se nas carótidas cervicais, normais. O ramo scguinte é a A. axilar direita, que se origina do contôrno lateral da aorta, distalmente ao tronco bicarótico. A A. axilar esquerda parte 1 polegada, distalmente ao vaso homônimo direito, tamóén do contôrno lateral da aorta, porém, acima do esôfago e da truquéia. O ligamento arterioso inicia-se na emergência da axilar esquerda, dirige-se ventral e algo caudalmente, à esquerda do esôfago e traquéia, para atingir a A. pulmonar. Tem cêrca de $3 / 4$ de polegada de comprimento por $1 / 10$ de polegada de espessura, sendo - Iume obliterado por completo.

Bossi (1909), entre os tratadistas, é o único a merecer especial refeJência, pois, afirma que as variedades do arco aórtico se observam com extrema raridade nos mamíferos domésticos, traduzindo-se por disposiçōes anormais ou origem diversa dos troncos provindos da convexidade do arco, acrescentando: diferenças de origem verificam-se notadamente nos carnivoros e roedores. Quanto an cão, esclarece que da convexidade aórtica podem nascer diretamente uma ou ambas as carótidas; em outras ocasiōes as duas axilares estāo reunidas na origem de modo a constituir tronco único. As variedades do areo aórtico nos mamiferos domésticos não tem, pela sua raridade, interêsse prático, mas apresentam importância morfológica, e são explicadas através os ensinamentos apreendidos a propósito da ontogenia, finaliza o $\mathrm{A}$.

Ėnfeixando esta série de observações qualificáveis, em sua maioria, como documentários de interêsse clínico a propósito de casos de estenose esofágica, condicionados pela situação inusitada da aorta à direita ocasionando o encarceramento do esôfago e da traquéia entre aorta, A. pulmonar e ligamento arterioso (GoRTon, JEX-BLAKE, MILKS, Yamamoto e Eмoto, Milks e Williams, Török, Möcsy, Davies e OTTAWAY) comprova-se que: 1) a ocorrência de anomalias do arco aúrtico e suas colaterais não constituem, no cão, fatos de caráter excepcional ; 2) a maior parte dos A. apenas se preocupou em averiguar as causas imediatas da obstrução esofágica, sem dar maior realce a importantes aspectos anatomicos, sem dúvida, injustificadamente omitidos.

JEX-Blake; DAVIES e OTTAWAY, entretanto. discutem seus casos sob o ponto de vista anatômico emprestando-lhes destaque sufi- 
ciente para que nos seja permitido ajuizar da disposiçāo das colaterais aórticas.

A leitura de parte do relato necroscópico subscrito por JEXBLAKE, cujo teor é transcrito na língua em que foi exarado, veicula equívoco, como já denunciára BRANDT, certamente atribuível a êrro de impressāo. De fato, os diagramas clássicos de BoAs, HochstetTer e as investigaçoos de Locy, Twining e LiLLie, além do esquema modificado de PoHLmas, nāo deixam dúvidas quanto à origem do arco da aorta nas aves: o $4 .^{\circ}$ arco desaparece inteiramente $\dot{a}$ esquerda, enquanto, o da direita persiste para constituir o Arcus aortae. O título de uma das publicações de PoHLMan (1920), por outro lado, esclarece em que condições se pode verificar a persistência do $4 .^{\circ}$ arco ártico à esquerda: "Consideration of the branchial arcades in chick based on the anomalous persistence of the $4^{\text {th }}$ left arch in a sixteen day stage".

Segundo afirma JEX-PLAKE ao expor sua observação, os ramos do arco aórtico, em número de 4 , partem isoladamente na seguinte ordem: A. carótida direita, A. carótida esquerda, A. subclávia direita e A. subclávia esquerda. Já DAvies e OtTAway descrevem disposição a 3 ramos, pois, a primeira colateral é representada por curto tronco bicarótico, ao qual se seguem, em ordem, A. axilar direita e A. axilar esquerda.

Mais interessantes são as observações de BRANDT. De fato, o A. aponta casos de arco aórtico direito dando origem a duas colaterais: A. braquincefálica e A. subclávia esquerda, tendo esta, para atingir o membro correspondente, percurso supra-esofágico. O A. considera muito raras tais anomalias vasculares, às quais se associa a constrição esofágica. BRANDT silencia, contudo, quanto ao comportamento dos Nn. laríngeos caudais.

Enfim, a ocorrência da A. subclávia direita como último ramo da crossá aórtica em mamíferos, parece nāo figurar entre as anomalias mais encontradiças. Conhecem-se apenas os trabalhos de Meckel e SMITI1, através a monografia de HOLZAPFEL, registrando-as respectivamente, no ouriço e no coelho. Também EALes notifica cssa anomalia no coelho atribuindo-lhe o qualificativo de rara. Graças ao esquema ilustrativo do caso, infere-se ser êste, em seus traços mais grosseiros, aquêle que mais se assemelha às observaçōes expostas a seguir, de modo especial no tocante ao comportamento do $\mathrm{N}$. laringeo caudal.

Para finalizar estes comentários, devemos esclarecer não ter encontrado em meio às variações dos troncos da convexidade da crossa aórtica nos carnivoros, referidas por LE DoubLe (1901), menção à 
ก. M. Paiva - A. subclávia direita como última colateral da atorta no cão 211

emergência da A. subclávia direita como última colateral aórtica, com trajeto supra-esofágico.

Parsons (1902), de outra parte, reportando-se às modificaçóes dos ramos aórticos, revela jamais ter visto nos mamíferos inferiores exemplo de $\mathrm{A}$. vertebral partindo do arco aórtico, como tamhém 0 da interessante anomalia do homem, na qual a $\mathrm{A}$. subclávia direita se origina como última colateral.

\section{OBSERVACÖES PESSOAIS}

(Hbscruccaso I - Fêmea adulta de talhe médio, mestiça de Foxterrier, correspondendo-lhe o n. ${ }^{\circ} 35$ na série de exemplares recolhidos para pesquisa da freqüência do Truncus bicaroticus. Procedência: Depósito da Prefeitura Municipal. Seguindo a téenica de rotina adotada para permitir apreciar a disposição dos ramos da crossa da aorta, no cào, scm especiais cuidados, pois, como se disse, era nosso objetivo o simples estudo da frequiência do tronco bicarútico, longe estávamos de imaginar a surpresa que nos aguardava ao preparar o exemplar n. ${ }^{\circ}$ 35 e essa inadvertência custou-nos, quando menos, a perda das relafooes ro canal torícico em seu tracto terminal. Diga-se de passagem, náo se empresta atualmente a mesma atenção, sucitada pelos apontamentos de S7AWLowsKY (1888), concerrientes à maneira de desembocar do canal torácico, quando a $A$. subclavia dextra emerge como última colateral aórtica.

A peça, disseccada de início a fresco, foi submetida tão logo quanto se percebeu a existência dá anomalia, a injeção intra-arterial de solução aquosa de formol a $2 \%$, seguida então de outra injeção, agora de gelatina corada com cinábrio. Completou-se a fixação da peça imergindo-a em solução aquosa de formol a $10 \%$.

No exemplar em aprêço o primeiro ramo a originar-se da crossa da aorta, a $39 \mathrm{~mm}$ do início do arco aórtico, è o Truncus bicaroticus. Este apresenta $4.1 \mathrm{~mm}$ de diâmetro c $45 \mathrm{~mm}$. de comprimento dando saída às $A a$. curotides communes sinistra e dextru, ambas de calibre (2.5 e $2.8 \mathrm{~mm}$ ) e decurso normais. Distalmente as tronco bicarótico, a $42 \mathrm{~mm}$ do ponto de emergência da aorta, nasce a $A$. sublelavia sinistru, com $4 \mathrm{~mm}$ de diâmetro, e, imediatamente, a seguir, a $A$. subclaviu dextra. Fayem-no, todavia, de tal forma, que a exame menos acurado chegam a dar a impressão de se originarem por tronco comum, pois, a A. subclávia direita situa-se crânio - e algo distalmente à sua homônima esquerda, entrando mesmo em contacto, cmbora muito restrito, com sua parede. Esta localização esclarece porque a $\mathrm{A}$. subclávia direitil é totalmente encoberta pela correspondente esquerda, quan- 
do se examina o contôrno convexo do arco aórtico, sem deslocar convenientemente a traquéia.

Os orifícios de abertura das Au. subclaviue, inspecionados através incisão praticada ao longo da concavidade da crossa aórtica, mostram-se separados por um esporão.

A A. subclávia direita, emergindo do contôrno cranial da aorta, dirige-se para a margem cranial dia $1 .{ }^{a}$ costela direita, abaixo da inserção do $M$. scalenus primae costae, com orientação a princípio dorsal e logo após oblíqua cranialmente, cruzando a linha módia à altura da articulação $7 .^{a}$ V. C. $-1 .^{a}$ V. T., dorsalmente ao esôfago e traquéia, entre estas formaçōes e o $M$. longus colli. O esôfago, desprovido de qualquer divertículo, e o $M$. longus colli mostram, nítida, a impressão do trajeto vascular. Leve-se em conta, entretanto, a irifluência da repleção do vaso pela massa corada e ulterior fixação da peça na produção de ditas marcas. Não existem, todavia, vestígios de estenose esofágica.

Desde a origem até a margem cranial da $1 .^{\mathrm{a}}$ costela, a A. subclávia direita apresenta $42 \mathrm{~mm}$ de comprimento, 5 e $4.6 \mathrm{~mm}$ de diâmetro, aquém c além do ponto em que se encontra compreendida entre esôfago e M. longo do pescoço, onde é exiguamente menos calibrosa $(4 \mathrm{~mm})$. Seus ramos colaterais, ao invés de partirem na ordem habitualmente assinalada, isto é, $A$. vertebralis, Truncus costocervicalis, Truncus omocervicalis, $A$. thoracica interna e $A$. thoracica externa. como sucede em relação à $A$. subclávia esquerda, mostram à seguinte alteração: o tronco costocervical é desmembrado, pois, a $A$. transversa colli parte isolada da A. subclávia direita, proximalmente ao tronco commum das Aa. cervicalis profunda e intercostalis suprema.

O N. recorrente, à esquerda, apresenta a disposição normal, por outras palavras, contorna o arco aórtico, junto ao ligamento arterioso, e inicia o trajeto retrógrado, em direção à laringe, aplicaclo ì face ventral e depois à face lateral da traquéia. Já à dircita, o N. recorrente não oferece as relações clàssicamente descritas, porquanto, dêsse lado o $\mathrm{N}$. vago não o fornece $\mathrm{em}$ sua porção torácica e conseqüentemente não se observa o giro que em condições normais aquêle nervo efetua ao redor do tracto inicial da A. subclávia direita ou da porção terminal da A. braquiocefálica, para entāo assumir direção recorrencial, até atingir a laringe.

O N. laríngreo caudal direito, origina-se da porção cervical do N. vago, aproximadamente so nível da articulação $4 .^{a}$ V. C. -- 5. ${ }^{\mathrm{a}} \mathrm{V}$. C.. formando com ôle ângulo de $25^{\circ}$; segue depois com trajeto rotrógrado. em direção cranial, para alcançar a laringe depois de $49 \mathrm{~mm}$ de percurso. 
O. M. Paiva - A. subclávia direita como última colateral da aurta no cão 213

Os gânglios nervosos cervical caudal e primeiro torácico estabelecem conexão através dois ramos dispostos de forma a abraçar a A. subclávia dircita em alça nervosa, a Ansa subclavia.

Em resumo, o N. laríngeo caudal apresenta, com efeito, trajeto recorrente, mas trajeto muito menos !ongo, pois, sua origem fazse em ponto bem mais alto, $\mathrm{cm}$ plena porção cervical do $\mathrm{N}$. vago. Como se demonstrará, aquêle ramo do $\mathrm{N}$. vago tem suas relações inteiramente alteradas em virtude da origem e decurso anomalos da A. subclávia direita.

Obsernarăo $1 /$ - Feto de têrmo, fêmea, retirado morto, juntamente com outros cinco irmãos, quatro machos e uma fêmea, mediante cesariana praticada em cadela mestiça de Iulu, no Departamento de Cirurgia da Faculdade de Medicina Veterinária, enviados por gentileza do Dr. Ernesto A. Matera. Ao exemplar coube o n. ${ }^{\circ} 66$, na série de animais recolhidos para estudo da frequiência do tronco bicarótico.

Quer pela presença do Truncus bicaroticus, quer pela origem, percurso e relaçōes da $A$. subclavia dextra, quer finalmente, pelo comportamento do $N$. laryngeus caudalis direito, oriundo, de modo análogo, da porção cervical do $N$. vagus, torna-se dispensável descrição pormenorizadar das anomalias vásculo-nervosas ocorrentes neste feto, pois, guardadas as devidas proporçōes, clas são essencialmente identificáveis às anotadas no caso 1. Merece, entretanto, particular reparo a anastomose pré-traqueial que se observa entre os $\mathrm{Nn}$. recorrente esquerdo e vago dircito, conexão qualificável como invulgar.

Consoante hábito em trabalhos relativos a anomalias dos grandes vasus originários da crossa aórtica, intentaremos também apresentar a interpretação embriogênica do achado anatômico. De resto, tal interpretação, proposta pela primeira vez por Wood (in BANCHI - 1907) tem sido enunciada, repetidas vêzes, em inúmeras publicaçôes da extensa literatura anatômica humana com respeito à emergência da A. subclávia direita como último ramo da crossa da aorta. Ao expô-la, pois, sumiriamente de novo, ampara-inos como melhor argumento, o interêsse em facê-la acompanhar a primeira publicação, cm nosso meio. no âmbilo da literalura veterinária, a registrar a anomalia $\mathrm{cm}$ estudo. Rememoremos. para tanto, de modo sucinto, as rases iniciais: do de:ienvoivimento do sistema arterial.

Ao esbòço do bulbo segue-se, cefìlicamente, o Truncus arteriosus o qual dá origem a dois ramos ascendentes, as aortas ventrais (Aa. 
sub-branquais); estas atingem a altura do $10^{\circ}$ arco branquial onde se infletem, dorsalmente, para constituir o $10^{\circ}$ arco aórtico. Já agora, como aortas primitivas dorsais (Aa. supra-branquiais) e com decurso descendente, os dois vasus, a princípio independentes, convergem : unem-se para formar a aorta comum (Aortn descendens).

As Aa. aortas ventrais e primitivas dorsais, anastomosadas através o $1 .^{\circ}$ aren ártico, reunem-se, com o progredir do desenvolvimento embrionário, por outros arcos dispostos transversal e paralelamente ao $10^{\circ}$; constituem-se, assim, em ordem crânio-caudal, o 2.", $3 .^{\circ}, 4 .^{\circ}, 5 .^{\circ}$ e $6 .^{\circ}$ arcos aórticos, o penúltimo dos quais tem, aliás, duriação efêmera e liga a aorta ventral à parte dorsal do 6." arco. A existência, embora não concomitante, dos seis arcos árticos, permite distinguir os segmentos intermediários, ou seja, os tractos arteriais situados entre as extremidades de dois arcos aórticos contígurs, tanto dorsal quanto ventralraente.

Por outro lado, no tracto que medeia entre o $6 .^{\circ}$ arco e o início da aorta comum, as aortas primitivas dorsais inrnecem, de ambos os lados, uma série de artérias intersegmentárias; à custa do 6. par de vasís intersegmentários constiłuil-se-än as Aa. subclavia. dextra e sinistra.

Passan-se a seguir transformações caracteri\%alas, em simples anáiise, pela atrofia de aiguns arcos aórticos e segmentos intermediários, ao rresmo tempo em que outros tantos, permanecendo, se desenvolvem desigualmonte; dêste modo, a simetria inicial cede lugar à acentuada assimetria observável no adulto. Com efeito: a) os $10^{\circ}, 20^{\circ}$ e 5." arcos aórticos atrofiam-se, totalmente, de ambos os lados; b) o $3 .^{\circ}$ arco e os $1 .^{\circ} \circ 2 .^{\circ}$ segmentos intermediários dorsais e ventrais têm sua persistência bilateral ligada à formação das Ait. carótilas inter-

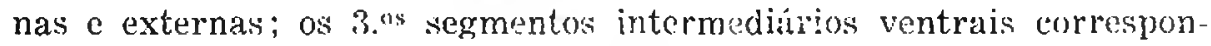
derão à Aa. carótidas comuns, os dorsais involvem inteiramente; c) os 4. arcos aúrticos têm destino diverso de cada lado: o da esquerda transformar-se-á no Arcus aortas, mantendo intima associação com a A. carotida primitiva cisquerda e continuando-se jella Aorifa descende'ns graças à permanência do 5." e 6." segmentos intermediários e tracto terminal da aorta primitiva dorsal esquerda: o da direita vira a representar o tronco braquiocefálico; d) as Au. subcluvicu, tendo emigrado cefàlicamente ao longo das aortas dorsitis, assumem, a da esquerda, a posição definitiva como ramo da crossa aórtica, enquanto, a da direita, com o desaparecimento da aurta primitiva dorsal homolateral. no trac dentre a $A$. subclávia clireita e aorta comum, passa a ter por base o $40^{\circ}$ arco aórtico direito; das Aa. subclávias partem, cefàlicamente, as Aa. vertebrais, ì guisa de anastomoses entre aque- 
las e os vasos segmentais mais craniais; e) o $6 .^{\circ}$ arco aórtico, ou pulmonar, à direita, só persiste em seu tracto justa-troncular para formar o ramo esquerdo da A. pulmonar; à esquerda, aumentando de calibre constituirá o Ductus arteriosus, pondo-se em continuidade no sua origem com a $\mathrm{A}$. pulmonar, no ponto de onde partirá a arteríola que virá a representar o ramo direito da A. pulmonar [CHIARUGI (1944 - pg. 43)]; a êsse tempo já o tronco arterial se desdobrou completamente e'm aorta sistemática e A. pulmonar; f) o ducto arterioso - comunicação entre A. pulmonar e porção descendente da d.orta - pérvio durante a vida fetal, atrofia-se após o nascimento, vindo a constituir o Ligamentum arteriosum, no adulto.

Atinge-se, assim, a disposição definitiva do homem, pois, vê-se partir da crossa aórtica, sucessivamente: 1) tronco comum das $A a$. subclavia dextra e carotis communis dextra; 2) A. carotis communis sinistra; 3) A. subclavia sinistra. Nos animais domésticos, contudo, observar-se-á ainda processo de concentração das colaterais aórticas que, no cão e no porco, leva à formação da $A$. brachincephalica (tronco comum das $A$ a. curotides communes sinistra, dextra e da $A$. subclavia dextra) e $A$. subclavia sinistra, como os dois únicos ramos aórticos. A confluência das colaterais aórticas vai ainda mais adiante nos bovinos e eqüinos, nos quais o Truncus bruchiocephalicus concentra todos aquêles ramos aúrticos.

Recordando o desenvolvimento dos arcos aórticos é-nos possivel deduzir o provável mecanismo embriogênico determinante do defeito em exame: o $4 .^{\circ}$ arco aúrtico, à direita, atrofiou-se inteiramente, enquanto a raiz direita da aorta primitiva dorsal persistiu para constituir a A. subclávia direita.

A vizinhança das duas Aa. subclávias e o trajeto supra-esofágico da direita são igualmente de fácil análise; para o último fato dêmos por conhecida a situação das aortas primitivas dorsais aplicadas à face ventral da coluna vertebral, dorsalmente ao esôfago. Quanto à aproximação das duas Aa. subclávias, invocamos para explicá-la o fenômeno da migração arterial (MaNNU - 1925), ou o da simples concentração dessas duas colaterais aúrticas, quiçá após deslocamento real ou aparente do coração e vasos da base em direção caudal. Se é que ambos cs mecanismo: não interferem simultâneamente. A formação do troncu bicarótico encontra sua razão de ser na própria origem anômala da A. subclávia direita.

A alteração das relaçóes do $\mathrm{N}$. laríngeo caudal, em casos de $\mathrm{A}$. subclávia direita como último ramo da crossa aórtica, é tão conhecida quanto a própria anomalia arterial, à qual está condicionada, embora tenha sido descrita menor número de vêzes pelo fato de alguns inves- 
tigadores terem cleixado de referir-se à disposição do N. laríngeo caudal; por outro lado, nem sempre a presença da $\mathrm{A}$. subclávia direita anômala se liga à não recorrência do $N$. laringeo caudal a partir da porçāo torácica do N. vago. Fm verdarle, anotaram-se no homem casos de recorrência ao redor das Aa. vertebral, lireoidéia inferior e, menos freqüentemente, da A. subclávia dircita anormal, como se pode exemplificar, com os casos respectivos de IIolzapfel, Moreira DA ROCHA (1926) e TRIAS PLJOL (1915).

Nos dois casos descritos, o N. laríngeo caudal vai diretamente à laringe, partindo da porção cervical do N. vago, após curto trajeto recorrente, isto é, sem contrair as rêlações habituais com a A. subclávia homolateral ou com a $\mathrm{A}$. braquiocefálica, depois de ser lornecido pela porção torácica do N. vago. Para entender in gênese da anomalia nervosa, forca é acompanhar algumas fases do desenvolvimento ontogenético, precisamente, aquelas em que sc constituem os arcos aórticos, segundo a série de fenômenos reportados pouco acima.

Neste estágio, os Nn. vagos transitam ventralmente aos citados arcos aórticos, fornecendo cada qual, ao nivel do $5 .^{\circ}$ par de arcos, o correspondenie N. laríngeo caudal. Com a regressiso do 5.0 par de arcos aórtices, êstes ramos dos Nn. vagos passam a caminhar, direção à laringe, caudalmente ao $4 .^{\circ}$ par de arcos, ainda sem apresentar decurso retrógrado, pois, atingem a laringe pràticamente com decurso transversal.

$O$ deslocamento do coração em direção caudal e o alongamento da região cervical, alongamento a que se encontra subordinado o maior ou menor trajeto recorrente do N. laríngeo caudal (BRENNER - 1883), esclarecem o restante. Enlaçados nos $40^{\mathrm{m}}$ arcos aórticos os Nn. laríngeos caudais afastam-se, gradualmente, do seu território de distribuição laríngeo até atingirem a situação definitiva, quando lhes assenta com justeza o nome de Nn. recorrentes. Ora, com a atrofia do $4 .^{\circ}$ arco da direita, nos casos de A. subclávia direita como última colateral da crossá aórtica, nāo está sujeito o $\mathrm{N}$. laríngeo caudal desse lado à "imposição biológica" (LACES - 1946), meramente mecânica, de se distanciar do território laríngeo, como sucederia em condiçōes normais. A disposição dos ramos do N. vago, face ao particular evoiver dos $4 .^{\circ}$, $5.0^{\circ} 6 .^{\circ}$ arcos a orticos e ao crescimento da regiäo cervical, em embrióes de Lhama e outros animais de pescoço longo (Camelidac), nos quais o N. recorrente apresenta peculiar comportamento (VON SCHUMACHER - 1906, KAJAVA - 1911 e ELZE - 1912), parece-nos contraprova favorível ìquela interpretação.

Numa ordem de consideraçôs diversas e com a qual desejamos encerrar a notificação dos dois casos de emergência da A. subclávia 
O. M. Paiva - A. subclávia direita como última colateral da a a

direita como última colateral aúrtica em cães, há ainda importante aspecto a ser clebatido: diz respeito à hereditariedade das variações e anomalias das coiaterais aúrticas.

Sabe-se, com eleito, que Giacomini (1882) ao dissecar alguns negros teve ensejo de identificar em mãe e filha a mesma anomalia da A. subclávia dircita. Aliás, segundo se pode apreciar, através o exame dá iiteratura humana, a emergència da A. subclávia direita como último ramo da crossa aórtica, parece incidir com freqüência mais elevada nos melanodermas.

Por outro lado, MILKs e WiLliaMs, anteriormente citados, jorcm cm relevo a procedência de três dos animais apresentando diverlículo esofágico determinalo pelo encarceramento do esofago entre A. pulmonar, aorta e ducto arterioso; provenientes do mesmo "kennel", dois deles eram filnos do mesmo macho.

Finalmente, EDMonds e SAWIN (1935), mediante dissecção do arco aórtico $\mathrm{em} 1900$ cuelhos de genealogia conhecida, revelaram além do tipo normal de ramificação (A), outros 14 tipos ou subtipos, dentre os quais dois mais comuns: $\mathrm{B}(18 \%)$ e $\mathrm{E}(1 \%)$. Estes últimos, diferindo do tipo A em relação à origem da $\mathrm{A}$. subclávia direita, pela freqüência mais alta com que ocorrem $\mathrm{cm}$ certas linhagens, indicam a matureza provàvelmente hereditária dos vários tipos. As proporções cic segregraçăo obtidas para os tipos $A, B$ e $\mathrm{E}$ são conformes á hipótese Mendeliana difatorial. Os AA. chamam a atenção para a existência de variaçōes comparáveis no homem.

Acreditamos que se venha a demonstrar a nature\%a hereditária de insuspeitas anomalias e variações das colaterais aórticas em cäcs, especialmente, se o acaso permitir identificá-las em animais vivos, cuja ascendência seja conhecida e cuja descendência possa vir a ser dirigilla com vistas à análise genética. De qualquer forma - e quanTo a êste ponto de vista estamos de pleno acôrdo com DE GARIS (1938)

-- o Arcus aorticus constitui cstrutura anatomica das mais apropriadas para estudos de variação, de simetria e de hereditariedade.

\section{RESUMO}

Nestá publicação, apresentamos o relato de dois raros casos de A. subclavia dextra como última colateral da crossa aórtica, encontracios, acidentalmente, ao curso de estudo sôbre a freqüência do Truncus bicaroticus no cão. A $A$. subcluvia dextru anormal emerge crânio-distalmente à sua homônima esquerda, tendo ao cruzar a linha mediana, a caminho do membro torácico, decurso supra-esofágico. Entre os scus ramos colaterais nota-se a seguinte alteração: o Truncus costocerpicalis apresenta-se desmembrado, partindo a $A$. tronisnerst colli isola- 
da e proximalmente ao tronco comum das Aa. cervicalis profunda e intercostalis suprema. Além das disposiçōes ora mencionadas, verifica-se, em ambos os exemplares, que a origem das Aa. carotides commu nes se faz por longo tronco comum e que o $N$. laryngeus caudalis é fornecido pela porção cervical do $N$. vagus, não contraindo, portanto, suas habituais relações com a $A$. subclavia dextra ou com a $A$. brachiocephalica e vindo alcançar a laringe depois de curto trajeto recorrencial. Não se observa sinal de estenose esofágica, no ponto em que a artéria cruza o esôfago.

Em contraposição ao que observa na literatura anatômica humana, na qual contam-se às centenas os relatos de emergência da $A$. subclavia dextra como último ramo do Arcus norticus, a bibliografia anátomo-comparativa oferece o registro da anomalia somente no ouriço e no coelho (MECKEL, SMITH, EALES), merecendo os achados o qualificativo de raros.

Chama-se a atenção para a virtual falta de pesquisas sistemáticas sôbre o comportamento dos ramos aórticos em anatomia comparativa, resumindo-se os escassos e dispersos trabalhos a propósito das anomalias do arco aórtico e scus ramos mais calibrosos no cāo; $\mathrm{em}$ tôdas as circunstâncias o interêsse das publicaçōes vinculava-se à obstrução esofágica ocasionada pelas anomalias existentes, explicando-se, assim, a omissão de importantes pormenores anatômicos.

As anomalias são descritas em fêmea, adulta, mestiça de Foxterrier e em feto de têrmo, fêmea, mestiça de Lulu. Tanto pela prèsença do Truncus bicaroticus quanto pela origem, percurso e relaçōes da $A$. subclavia dextra e, finalmente, pelo comportamento do $N$. laryngeus cuudalis direito, às observações, essencialmente identificáveis, pode ser atribuida descrição comum. Apenas, deve ser destacada a anastomose pré-traqueial que se nota no caso II (feto), entre os $\mathrm{Nn}$. laryngeus caudalis esçuerdo e vagus direito, conexão de caráter invulgar.

Prosseguindo apresentam-se as explicações das anomalias vascular e nervosa, analisando-se as condiçōes embriológicas que encaminharam o desenvolvimento de ambas; conclui-se admitindo a possibilidade de demonstração da natureza hereditária de anomalias e variações das colaterais aórticas em cāes, tendo por base as contribuições de Giacomini (homem), Milks e Williams (cão) e Enmonds e Sawin (coelho).

De qualquer forma, e em consonância com o ponto de vista de DE GARIS, afirma-se que o Arcus aorticus constitui estrutura anatômica das mais adequadas para estudos de variação, de simctria e de hereditariedade. 
o. M. Paiva - A. subclávia direita como última colateral da aorta no cão 219

\section{SUMMARY}

This note gives the description of two cases of right subclavian artery ( $A$. s ub clavia dextra) as the last branch of the aortic arch, in the dog, found occasionally wiven the A. was studying the frequency of the Truncus bicaroticus.

The abinormal right subclavian artery arises cranio-distally to the left subclavian artery (A. subclavia sinistra), running towards the thoracic limb, above the oesophagus. Among its collateral branches is notod the following alteration: the transverse artery of the neck (A. transversa colli), instead of emerging as usually by a common trunk with the deep cervical artery (A. cervicalis prof $u n d a$ ) and supreme intercostal artery (A. intercostalis suprema), arises from the right subclavian artery isolated and proximally to the common stem of these two vessels. Besides these particular dispositions, both animals present the common carotid arterics (Aa. carotides communes) arising by a common long trunk (Truncus bicaroticus) and the right recurrent nerve (N. laryngeus caudalis) as a cervical branch of the vagus nerve $(N . v a g u s)$, i. e., without winding around either the right subclavian artery or the brachiocephalic artery (A. brachiocep $h a-$ lic (1) an therefore showing its recurrencial traject shorter than commonly. The oesonhagn's is not constricted at the arterial crossing place.

While human anatomical literature points out hundreds of cases of right subclavian artery as the last branch of the artic arch, the comparutive anatomical bibliogrephy records the anomaly only in the hedgehog and in the rabbit (NECKEL, SMITH, HALES) as very rare findings.

Attention is called to the virtual lack of sisterizatic researchs about the aortic collateral branches in comparative anatomy; the scanty and scattered cases referring to anomalies of the aortic collateral branches, in the dog, are snmmed $u p$. In all these cases the greatest interest uas ascribed to the constriction of the oesophagus; thus cun be explained the omission of imporlant analomical details.

The anomalies are described in tuo mongrel females: one is ainlt and the other a foetus at birth time. Botho the presence of the $T r u n c u s$ bicaroticus and the arising, course and relations of the right subcluvinu artery, besiles the behaviour of the right recurvent neve, permit a single description of the two cases. In the sccom? one (foctus), however, it is worthy to note an unusual annslomotic branch that connects the left recurrent and right vagus nerves. 
Explanations of the vascular and nervous anomalies, under the embryological standpoint, are given; the possibility of demonstrating the hereditary nature of anomalies and variations of the aortic collateral branches in dogs, in the light of GIACOMINI (human), MILKS and Williams (dog), EDMONDS and SAwIN (rubbit) findings, is considered admissible.

Amyray, according to DE GARIS, it is asserted that the Arcus aorticus corresponds to the most filling anatomical structure for variation, symmetry and heredity studies.

\section{BIBLIOGRAFIA}

BANCII, A. - 1907 - Il V arco uortico-branchiale nella interpretazione di aleune varictá dell'arco dell'aorta e dei suoi rami. Arch. Ital. Anat. Fmbriol., 6 (2) : $389-427$

Bossi, V. - 1909 (pref.) - "in" Bossi-Caradonna. Trattato di Anatomia Veierinatia. 2: 77. Milano, Finecsco Vallardi

BrandT, A. J. - 1940 - Rechtsseitige Aortabogen mit abnormen Verlauf der "A. subclavia sinistra" als Ursache von Oesophagusstenose beim Hund. Skand. Vet.: 993-1005, 1940 "in" Collected I'aprers Vet. Irest., Stoclinolm

CAIRNeY, J, - 1925 - The anomalous right subelavian artery considered in the light of recent findings in arterial development; with a note on two cases of an unusual relation of the innominate artery to the trachea. Jour. Anat., 59 (3) : 205-96

Chiarugr, G. - 1914 - Trattato di Embriologia. 4 (2). Milano, Societá Fditrice Libraria

DAvies, J. J. - OtTAWAY, C. W. - 1943 - A peculiar case of oesophageal obstruction in the dog: congenital abnormality of the aortic arch. Vet. Kece, 55 (8) : $102-3$

DF Gris, C. - 1938 - Branches of the aortic arch in 153 rhesus monkeys (second series). Anat. Fec., 70 (3): 251-62

Eales, N. B. - 1930 - Note on an abnormality in the blood vascular system of the rabbit. Jour. Anat, 65 (1): 152-3

Edmonds, II. W. - SAWin, P. B. - 1825 - Variations of the branches of the aortic arch in rabbits. Am. Nat., 70 (726): 48-9)

ELze, C. - 1912 - Nervus laryngeus inferior des Lamas (Auchenia lama). Anat. Anz., 12 (16): 410-4

Giacomini, C. - 1882 - Annotazioni sopra l' anatomia del negro. Seconda memoria, Torino, Tip. Cclansa

Holzaples, G. - 1899 - Ungewöhnlicher Ursprung und Verlauf der Arteria Subclavia dextia. Anut. Hefte, I Abt., I1. 40; 12 (3) : 369-i,2:t

JEX-BLAKE, A. J. - 1926 - Obstruction of the esophagus caused by a persistent "Ductus Arteriosus". Lancet, 211 542-14

Jores, I. - 1924 - "in" Henke, F. -- Lubarseh, O. - Handbuch der speziellen pathologischen Anatomic und Ilistologic. 2: Herz und Gefässe. Berlin, Julius Springer

KAJAVA, Y. - 1911 - Nervus vagus und Arcus aortae bei Lama. Anat. Anz., 40 (10) : $265-79$ 
O. M. Paiva - A. subclávia direita como última colateral da aorta no cão 221

LAGES, L. - 1946 - Nervo laríngeo inferior não recurrente e arteria subclávia direita retro-esofágiana (Importância desta anomalia do ponto de vista

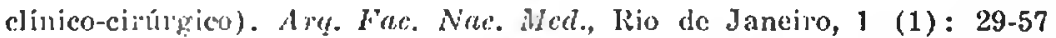

LE Dourle, A. F. - 1901 - Des variations des trones de la convexité de la crosse de l'aorte de l'homme e du gibbon, des singes cquadrupedes et des carnas-

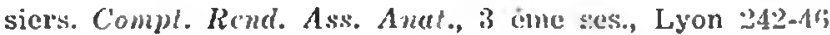

Mannu, A. - 1925 - Come si deve intendere es spicgare la migrazione o movimento reale delle radice arleriose. Monit. Zool. Ilal., ibj: 147-54

Mirks, IH. J. - 1929) - Diverticulum of the esophagus due to congenital malformation. Vet. $M c d$, 21: $22 \%-8$

Milks, H. J. - WILlians, W. J. - 1937 - l'ersistence oi the right instead of the left primitive aurta in the dog, incarcerating the esophagus and causing its dilatalion, Re?. Niew York sta. Vet. Coll., 1935-6: 51-4

Möcsy, J. von - 1939 - Two cases of oesophageal strangulation (Trans. title) Tierärztl. Rdsch., 45: 34:3-5 "in" Vel. Rec., 32 (23) : 431

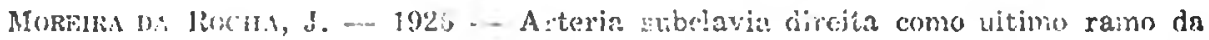
crossa antica. An. Fuc. Med., S. Puulo, 1: 103-2.t

PARSoNs, F. G. - 1902 - On the arrangement of the branches of the mammalian aortic arch. Jour. Anat. and Physiol, 36: 387-99

Pollowan, A. G. - 1920 - A consideration of the branchial arcades in chick based on the anomalous persisicnce of the 4 th lef 6 arch in a sixteen day stuge. Auret. lace, 18 (2): 15i)-6G

Schumacher, S. von - 1906 - Über die Kehliopfnerven beim Lama (Auchenia l:ula) Lad Vicunna (Auchenia vicunna). Anut. Ani., 28 (5-6): $156-60$

S7Awlowsk, J. - 1888 - tiber das Verhalten des Ductus Thoracieus bei Fersisten\% der Rechten sbsteigenden Aorlenwurzel. Anat. Anz., 3 (27-28): $839-49$

Triaz Pujol, J. - 1915 - Un caso de anomalia de la arteria subelavia derecha y su explicación enibriogénica. Arch. Ancit. Anthropol., 3 (2): 17-27 


\section{EXPLICAÇĀO DAS FIGURAS}

Fig. 1 - Caso I: Fêmea, adulta, mestiça de Fox-terrier. Fig. 2 - Caso II: Fêmea, feto de têrmo, mestiça de Lulu.

Legenda:

A. c. c. d. - A. carótida comum direita.

A. c. c. e. - A. carótida comum esquerda.

A. s. d. s. - A. subclávia direita supra-esofágica.

A. s. e. - A. subclávia esquerda.

T. b. - Tronco bicarótico.

N. v. d. - N. vago direito.

N. v. e. - N. vago esquerdo

N. 1. c. e. - N. laríngeo caudal esquerdo

r. p. a. - ramo anastomótico pré-traqueial 

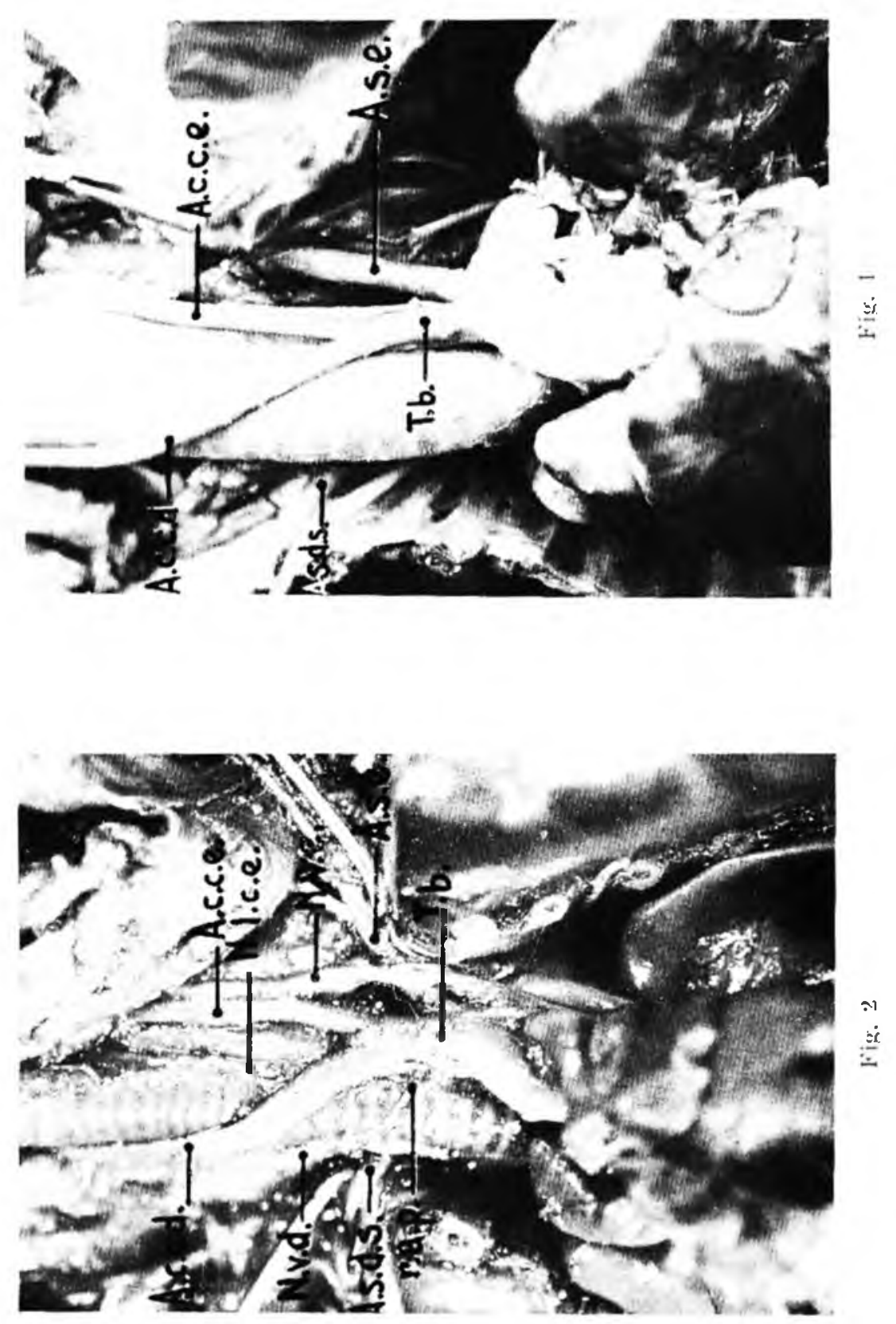